\begin{tabular}{|c|l|}
\hline Title & Multichannel dynamical symmetry and cluster-coexistence \\
\hline Author(s) & Cseh, J.; Katō, $\mathrm{K}$. \\
\hline Citation & $\begin{array}{l}\text { Physical Review C, 87(6), 067301 } \\
\text { https://doi.org/10.1103/PhysRevC.87.067301 }\end{array}$ \\
\hline Issue Date & 2013-06-05 \\
\hline Doc URL & http://hdl.handle.net/2115/63072 \\
\hline Rights & ○2013A merican Physical Society \\
\hline Type & article \\
\hline File Information & PhysRevC87 067301.pdf \\
\hline
\end{tabular}

Instructions for use 


\title{
Multichannel dynamical symmetry and cluster-coexistence
}

\author{
J. Cseh \\ Institute for Nuclear Research, Hungarian Academy of Sciences, Debrecen, Postafiók 51, Hungary 4001 \\ K. Katō \\ Nuclear Reaction Data Centre, Faculty of Science, Hokkaido University, Sapporo 060-0810, Japan
}

(Received 23 January 2013; published 5 June 2013)

\begin{abstract}
A composite symmetry of the nuclear structure, called multichannel dynamical symmetry, is established. It can describe different cluster configurations (defined by different reaction channels) in a unified framework; thus, it has a considerable predictive power. The two-channel case is presented in detail, and its conceptual similarity to the dynamical supersymmetry is discussed.
\end{abstract}

DOI: 10.1103/PhysRevC.87.067301

PACS number(s): 21.60.Fw, 21.60.Gx

Symmetry considerations can simplify the solution of complex problems to a large extent. In particular, they turned out to be very useful in the study of nuclear structure, too. Symmetries of a special kind, which we call here composite symmetries, give a unified description of systems of coupled degrees of freedom. Notable examples in nuclear physics are the pioneering supermultiplet theory by Wigner [1], accounting for proton and neutron degrees of freedom, and more recently the dynamical supersymmetry (SUSY) models, describing the collective motion and the single nucleon degrees of freedom [2-4].

The coexistence of different cluster configurations in a nucleus is an interesting example of a system of coupled degrees of freedom. Remarkable examples are the ${ }^{12} \mathrm{C}+{ }^{12} \mathrm{C}$ and ${ }^{20} \mathrm{Ne}+{ }^{4} \mathrm{He}$ configurations in ${ }^{24} \mathrm{Mg}$, or the ${ }^{12} \mathrm{C}+{ }^{16} \mathrm{O}$ and ${ }^{24} \mathrm{Mg}+{ }^{4} \mathrm{He}$ configurations in ${ }^{28} \mathrm{Si}$. In Ref. [5] the multichannel dynamical symmetry (MUSY) was introduced to describe this phenomenon. Here the channel refers to the reaction channel, which defines a binary cluster configuration. The idea was invented at the phenomenological level, based on general physical arguments, which resulted in relations for the energy eigenvalues [5]. However, the mathematical background, and the exact physical nature of this new symmetry, has not been revealed so far. Here we present the scenario of how one can establish the algebraic structure of the MUSY in general, and we give a detailed derivation for the two-channel dynamical symmetry.

To illustrate the main features of this new symmetry it seems to be proper to recall some basic vocabulary on symmetries. A continuous symmetry is an exact one if the Hamiltonian commutes with the generators of its Lie group. A dynamical symmetry is said to hold if the Hamiltonian can be expressed in terms of the invariant operators of a chain of nested subgroups (see, e.g., Refs. [6-8]): $\mathrm{G} \supset \mathrm{G}^{\prime} \supset \mathrm{G}^{\prime \prime} \supset \cdots \supset \mathrm{G}^{f}$. (Sometimes this symmetry is called a dynamically broken symmetry, because only $\mathrm{G}^{f}$ is an exact symmetry.) In such a case the eigenvalue problem of the energy has an analytical solution, and the labels of irreducible representations are good quantum numbers.

Let us consider a system of two components ( 1 and 2), each of them described by an algebraic model and having (at least one) dynamical symmetry: $\mathrm{G}_{i} \supset \mathrm{G}_{i}^{\prime} \supset \mathrm{G}_{i}^{\prime \prime} \supset \cdots ; i=1,2$. If the particle numbers of the two components are conserved separately, then the algebraic model with group structure $\mathrm{G}_{1} \otimes$ $\mathrm{G}_{2}$ usually proves to be a successful approach. The subgroups of $G_{1} \otimes G_{2}$ define the relevant dynamical symmetries of the system.

Deeper symmetries, with different nature, arise from the embedding of the direct product group into a larger group: $\mathrm{G}_{0} \supset \mathrm{G}_{1} \otimes \mathrm{G}_{2}$. Some generators of $\mathrm{G}_{0}$ transform particles of type 1 into particles of type 2 , or vice versa. In this article we refer to a composite symmetry in this sense. In the supermultiplet scheme of Wigner, e.g., the protons and neutrons are not conserved separately $[1,3]$. In the SUSY models collective phonons and nucleons are transformed into each other because of the embedding into graded Lie algebras $[\mathrm{U}(6 / \mathrm{m})$ $\supset \mathrm{U}(6) \otimes \mathrm{U}(m)$ in the quadrupole [2,3], and $\mathrm{U}(4 / m) \supset$ $\mathrm{U}(4) \otimes \mathrm{U}(m)$ in the dipole, i.e., cluster models [4]].

The multichannel dynamical symmetry is formulated in the framework of the semimicroscopic algebraic cluster model (SACM) [9], in which the clusterization of atomic nuclei is described in a fully algebraic way. The model space is constructed microscopically; thus, one can take into account that the antisymmetrization may wash out the difference between different cluster configurations.

The logic of the MUSY, illustrated by the two-channel case, is as follows:

(i) First we show that the two binary clusterizations are related to each other by an underlying ternary configuration, which has two different sets of the relevant Jacobi coordinates.

(ii) The transformation between the two sets is well established and has definite algebraic structure (obtained by the extension of the results of Refs. [10-13]).

(iii) A chain of nested subgroups enables us to define a dynamical symmetry of the ternary configuration.

(iv) The two-channel dynamical symmetry of the different binary configurations is obtained from the dynamical symmetry of the ternary configuration by projection.

This rigorous derivation results in the same energy functional, which was obtained in a phenomenological way in Ref. [5].

In what follows, first we recall the basic features of the SACM and the empirical introduction of the MUSY, then we go step-by-step in its rigorous derivation. The example we consider involves the ${ }^{16} \mathrm{O}+{ }^{8} \mathrm{Be}$ and ${ }^{20} \mathrm{Ne}+{ }^{4} \mathrm{He}$ configurations of ${ }^{24} \mathrm{Mg}$, in which case each cluster of the underlying ternary 
configuration $\left({ }^{16} \mathrm{O}+{ }^{4} \mathrm{He}+{ }^{4} \mathrm{He}\right)$ is $\mathrm{SU}(3)$ scalar. Finally we compare some characteristic features of this new symmetry to those of the dynamical supersymmetry.

The semimicroscopic algebraic cluster model [9] describes the internal structure of the clusters by the Elliott model [6]; therefore, its wave function has a $U_{C}^{S T}(4) \otimes U_{C}(3)$ symmetry, where $\mathrm{C}$ indicates a cluster. The relative motion of the clusters is accounted for by the modified vibron model [14]. This is an algebraic model of the rotation and vibration of a two-body system in the three-dimensional space, which has a $U_{R}(4)$ group structure ( $\mathrm{R}$ stands for relative motion). The spin and isospin degrees of freedom are essential in constructing the model space. However, if one is interested only in a single supermultiplet $\left[\mathrm{U}_{\mathrm{C}}^{\mathrm{ST}}(4)\right]$ symmetry, which is typical in cluster problems, then from the viewpoint of the operators the relevant group structure simplifies to that of the space part:

$$
\begin{aligned}
& \mathrm{U}_{\mathrm{C}}(3) \otimes \mathrm{U}_{\mathrm{R}}(4) \quad \supset \mathrm{U}_{\mathrm{C}}(3) \otimes \mathrm{U}_{\mathrm{R}}(3) \quad \supset \quad \mathrm{U}(3) \quad \supset \mathrm{SU}(3) \supset \mathrm{SO}(3) \supset \mathrm{SO}(2) \\
& \left|\left[n_{1}^{\mathrm{C}}, n_{2}^{\mathrm{C}}, n_{3}^{\mathrm{C}}\right],\left[N_{\mathrm{R}}, 0,0,0\right], \quad\left[n_{\mathrm{R}}, 0,0,\right], \quad\left[n_{1}, n_{2}, n_{3}\right],(\lambda, \mu), K, \quad L, \quad M\right\rangle .
\end{aligned}
$$

The coupled $\mathrm{U}(3)$ group is generated by the $n=n_{\mathrm{C}}+n_{\mathrm{R}}, Q=$ $Q_{\mathrm{C}}+Q_{\mathrm{R}}$, and $L=L_{\mathrm{C}}+L_{\mathrm{R}}$ operators, where $n$ is the particle (quantum) number operator, $Q$ is the quadrupole momentum, and $L$ is the angular momentum.

This strong-coupled $\mathrm{U}(3)$ basis is especially useful for treating the exclusion principle [9], because the U(3) generators commute with those of the permutation group [15]. The exclusion of the Pauli-forbidden states is a modification [9] with respect to the original vibron model, as it is applied, e.g., in molecular physics [16].

The argumentation of Ref. [5] introducing the MUSY on the level of eigenvalues is as follows. Let us consider two different binary clusterizations of a nucleus. For the sake of simplicity let each cluster be spin-isospin scalar, and let one cluster in both configurations be SU(3) scalar (having closed shell structure). An example is the ${ }^{20} \mathrm{Ne}+{ }^{4} \mathrm{He}$ and ${ }^{16} \mathrm{O}+$ ${ }^{8} \mathrm{Be}$ configurations of the ${ }^{24} \mathrm{Mg}$ nucleus. What is the relation between the energy spectra of the two configurations, if the dynamical symmetry (1) holds for both of them? The U(3) basis states of the different clusterizations are not orthogonal to each other; they may have considerable overlap as a consequence of the antisymmetry of the wave function. In some cases the two wave functions can even be identical to each other. Therefore, it is natural to require that their energies be the same, too. This requirement establishes a relation between the energy eigenvalues of the two Hamiltonians. In Ref. [5] the energy functional

$$
E=\epsilon+\gamma n_{\mathrm{R}}+\beta L(L+1)+\theta n_{\mathrm{R}} L(L+1)+F(\lambda, \mu, L)
$$

was applied for the spectra of two different $(c$ and $d$ ) binary clusterizations with the constraints of $\gamma_{c}=\gamma_{d}=\gamma$, $\epsilon_{c}=\epsilon_{d}+\gamma n_{0}, \theta_{c}=\theta_{d}=\theta, \beta_{c}=\beta_{d}+\theta n_{0}, F_{c}(\lambda, \mu, L)=$ $F_{d}(\lambda, \mu, L)$, and ${ }_{c} n_{\mathrm{R}}={ }_{d} n_{\mathrm{R}}+n_{0}$, where $n_{0}$ is the difference of the relative motion quantum number in describing the same $\mathrm{SU}(3)$ state.

The microscopic foundation of the MUSY starts with the introduction of an underlying multicluster configuration. For the two-channel case it is a ternary configuration. We consider two different binary clusterizations, $c: C_{1}+C_{2}$ and $d: D_{1}+D_{2}$, and suppose that the relations of the mass numbers are as follows: $A_{C_{1}} \geqslant A_{C_{2}}, A_{D_{1}} \geqslant A_{D_{2}}, A_{D_{1}} \geqslant A_{C_{1}}$, and $A_{C_{2}} \geqslant A_{D_{2}}$. (In the example of the ${ }^{24} \mathrm{Mg}$ nucleus, $C_{1}$ : ${ }^{16} \mathrm{O}, C_{2}:{ }^{8} \mathrm{Be}, D_{1}:{ }^{20} \mathrm{Ne}, D_{2}:{ }^{4} \mathrm{He}$.) Let us consider the fol- lowing ternary fragmentation: $\left(C_{1}\right)+(C D)+\left(D_{2}\right),(C D)=$ $\left(C_{2}-D_{2}\right)=\left(D_{1}-C_{1}\right)$. (In the example, $\left.{ }^{16} \mathrm{O}+{ }^{4} \mathrm{He}+{ }^{4} \mathrm{He}.\right)$ Another ternary clusterization is given by $\left(C_{2}\right)+(D C)+$ $\left(D_{2}\right), \quad(D C)=\left(C_{1}-D_{2}\right)=\left(D_{1}-C_{2}\right)$. (In the example, ${ }^{8} \mathrm{Be}+{ }^{12} \mathrm{C}+{ }^{4} \mathrm{He}$. $)$

We suppose that each fragment has a definite U(3) symmetry (intrinsic state), and we consider the ternary fragmentation, which is simpler in the sense that it contains more SU(3)-scalar clusters, e.g., $\left(C_{1}\right)+(C D)+\left(D_{2}\right)$. Then the two sets of Jacobi coordinates defining the binary configurations are as follows: $\mathbf{t}_{c}=\mathbf{r}_{D_{2}}-\mathbf{r}_{C D}, \mathbf{s}_{c}=\mathbf{r}_{C_{1}}-\left(M_{D_{2}} \mathbf{r}_{D_{2}}+\right.$ $\left.M_{C D} \mathbf{r}_{C D}\right) /\left(M_{D_{2}}+M_{C D}\right)$, and $\mathbf{t}_{d}=\mathbf{r}_{C_{1}}-\mathbf{r}_{C D}, \quad \mathbf{s}_{d}=\mathbf{r}_{D_{2}}-$ $\left(M_{C_{1}} \mathbf{r}_{C_{1}}+M_{C D} \mathbf{r}_{C D}\right) /\left(M_{C_{1}}+M_{C D}\right)$, where $M$ is the mass and $\mathbf{r}$ is the space vector of the corresponding cluster. Obviously, the clusterization $C_{1}+C_{2}$ corresponds to the coordinate set $c$ with some restriction on $\mathbf{t}_{c}$, while clusterization $D_{1}+D_{2}$ corresponds to the coordinate set $d$ with some restriction on $\mathbf{t}_{d}$.

The transformation from the clusterization $C_{1}+C_{2}$ to that of $D_{1}+D_{2}$ requires a transformation between the two sets of Jacobi coordinates, $\mathbf{s}_{c}, \mathbf{t}_{c}$ and $\mathbf{s}_{d}, \mathbf{t}_{d}$.

For the description of the two independent relative motions along the $\mathbf{s}$ and $\mathbf{t}$ vectors we introduce two sets of oscillator quanta (i.e., $l=1$ bosons). The corresponding creation operators ${ }_{i} \pi_{\mu}^{\dagger}(\mu=-1,0,1, i=1,2)$, and annihilation operators ${ }_{i} \pi_{\mu}$, satisfy the commutation relations $\left[{ }_{i} \pi_{\mu},{ }_{j} \pi_{\nu}^{\dagger}\right]=\delta_{i j} \delta_{\mu \nu}$. Here 1 and 2 refer to the sets of quanta along the $\mathbf{s}_{k}$ and $\mathbf{t}_{k}$ $(k=c, d)$ coordinates. Furthermore, an $(l=0)$ scalar $\sigma$ boson is introduced in order to be able to generate the spectrum [17]. The particle-number-conserving bilinear products, coupled to good spherical tensors, are

$$
\begin{aligned}
& { }_{i i} B_{m}^{(l)}(1,1)=\left[{ }_{i} \pi^{\dagger} \times{ }_{i} \tilde{\pi}\right]_{m}^{(l)}, \quad i=1,2, \\
& { }_{12} B_{m}^{(l)}(1,1)=\left[{ }_{1} \pi^{\dagger} \times \widetilde{\pi}\right]_{m}^{(l)}, \quad{ }_{21} B_{m}^{(l)}(1,1)=\left[{ }_{2} \pi^{\dagger} \times \widetilde{1}_{1}\right]_{m}^{(l)}, \\
& { }_{0 i} B_{m}^{(l)}(0,1)=\left[\sigma^{\dagger} \times{ }_{i} \tilde{\pi}\right]_{m}^{(l)}, \quad{ }_{i 0} B_{m}^{(l)}(1,0)=\left[{ }_{i} \pi^{\dagger} \times \widetilde{\sigma}\right]_{m}^{(l)}, \\
& { }_{00} B_{0}^{(0)}(0,0)=\left[\sigma^{\dagger} \times \widetilde{\sigma}\right]_{0}^{(0)} \text {. }
\end{aligned}
$$

Here ${ }_{i} \widetilde{\pi}_{\mu}=(-1)^{(-\mu)} \pi_{\mu}, \quad \tilde{\sigma}=\sigma$, and the square brackets indicate angular momentum coupling. The $2 \times 9$ operators of the first line generate two U(3) groups. Together with the other $2 \times 9$ operators of the second line, they generate a U(6) group. With the $2 \times 6+1$ operators containing (also) $\sigma$ bosons the $\mathrm{U}(7)$ group is obtained.

This group has a rich structure of subgroups; therefore, several dynamical symmetries can be constructed. The 
important one (for the present purpose) starts with the U(7) $\supset \mathrm{U}(6)$ group chain and contains a unified U(3) group and a pseudospin group $\mathrm{U}_{p}(2)[11,12]$. Group $\mathrm{U}(3)$ is generated by the operators $B_{m}^{(l)}(1,1)={ }_{11} B_{m}^{(l)}(1,1)+{ }_{22} B_{m}^{(l)}(1,1)$, while the $\mathrm{U}_{p}(2)$ generators are

$$
\begin{aligned}
& S_{-}^{p}=\sum_{m}{ }_{2} \pi_{m}^{\dagger}{ }_{1} \tilde{\pi}_{m}, \quad S_{+}^{p}=\sum_{m}{ }_{1} \pi_{m}^{\dagger}{ }_{2} \tilde{\pi}_{m}, \\
& S_{0}^{p}=\frac{1}{2} \sum_{m}\left({ }_{1} \pi_{m}^{\dagger}{ }_{1} \tilde{\pi}_{m}-{ }_{2} \pi_{m}^{\dagger}{ }_{2} \tilde{\pi}_{m}\right), \\
& N_{6}=\sum_{m}\left({ }_{1} \pi_{m}^{\dagger}{ }_{1} \tilde{\pi}_{m}+{ }_{2} \pi_{m}^{\dagger}{ }_{2} \tilde{\pi}_{m}\right)=n_{1}+n_{2} .
\end{aligned}
$$

Here $\sum_{m}{ }_{i} \pi_{m}^{\dagger}{ }_{j} \tilde{\pi}_{m}=\sqrt{3}_{i j} B_{0}^{(0)}(1,1)$. The pseudospin operators, first introduced by Bargman and Moshinsky [10] in a different context, act in the cluster-index space [13], and an exact $\mathrm{U}_{p}(2)$ symmetry means complete invariance with respect to all the transformations in the particle index space, including the finite rotation from the set of Jacobi coordinates $c$ to that of $d$.

The dynamical symmetry of the ternary configuration is described by the following group chain:

$$
\begin{aligned}
& \mathrm{U}(7) \supset \mathrm{U}(1) \otimes \mathrm{U}(6) \supset \mathrm{U}(6) \supset\{\mathrm{U}(3) \supset \mathrm{SU}(3) \supset \mathrm{SO}(3) \supset \mathrm{SO}(2)\} \otimes\left\{\mathrm{U}_{p}(2) \supset \mathrm{SU}_{p}(2) \supset \mathrm{SO}_{p}(2)\right\} \\
& \left|N_{7}, \quad n_{\sigma} \quad N_{6}, \quad\left[h_{1}, h_{2}, 0\right],(\lambda, \mu), K, \quad L, \quad M, \quad\left[h_{1}, h_{2}\right], \quad j_{p}, \quad m_{p}\right\rangle \text {. }
\end{aligned}
$$

The relations of the representation labels are as follows: $N_{6}=N_{7}, N_{7}-1, N_{7}-2, \ldots, N_{0} ; \quad h_{1} \geqslant h_{2}, \quad h_{1}+h_{2}=$ $N_{6}=n_{1}+n_{2} ; j_{p}=\frac{1}{2}\left(h_{1}-h_{2}\right) ; m_{p}=j_{p}, j_{p}-1, \ldots,-j_{p}$; $m_{p}=\frac{1}{2}\left(n_{1}-n_{2}\right) ; \quad\left[h_{1}, h_{2}\right]=\left[n_{1}+n_{2}, 0\right],\left[n_{1}+n_{2}-\right.$ $1,1], \ldots,\left[\max \left\{n_{1}, n_{2}\right\}, \min \left\{n_{1}, n_{2}\right\}\right] ; \quad \lambda=h_{1}-h_{2}$, $\mu=h_{2} ; \quad K=\min \{\lambda, \mu\}, \min \{\lambda, \mu\}-2, \ldots, 1 \quad$ or 0 ; if $K=0: L=\max \{\lambda, \mu\}, \max \{\lambda, \mu\}-2, \ldots, 1 \quad$ or 0, if $K \neq 0: L=K, K+1, \ldots, K+\max \{\lambda, \mu\} . \quad N_{0} \quad$ is the lowest Pauli-allowed value.

Omitting the redundant labels as well as $N_{7}$, which is taken to be a constant for a system, the basis states can be denoted in different ways: $\left|N_{6},\left[h_{1}, h_{2}\right], m_{p}, K, L\right\rangle$, $\left|N_{6}, m_{p},(\lambda, \mu), K, L\right\rangle,\left|n_{1}, n_{2},(\lambda, \mu), K, L\right\rangle$. This latter notation coincides with that of Ref. [13].

Note that the pseudospin operators are U(3) つ SU(3) つ $\mathrm{SO}(3)$ scalars, while the generators of $\mathrm{U}(3)$ are scalars with respect to the pseudospin. Therefore, these latter ones are invariant under the transformations in the cluster-index space.

The transformation of the building block (creation and annihilation) operators, between the different sets of Jacobi coordinates, can be found in Ref. [13]. They determine also the transformation of other physical quantities. The basis states of one set of coordinates can be expressed as a finite combination of the other basis, with the Talmi-Moshinsky coefficients. Therefore, the expectation values and overlaps expressed in terms of coordinates $c$ or $d$ can be transformed into each other. We leave these general investigations for a separate study; here we concentrate on the consequences of the dynamical symmetry.

A simple yet realistic Hamiltonian can be written as

$$
H=\epsilon+\gamma N_{6}+\delta C_{\mathrm{SU}(3)}^{(2)}+\beta L^{2}+\theta N_{6} L^{2},
$$

which is diagonal in the basis above, describing a dynamical symmetry of the underlying ternary configuration. Of course, more complicated functional forms of the invariant operators of U(3) and its subgroups can be applied, too, still having the dynamical symmetry. These Hamiltonians have an exact pseudospin symmetry.
The same procedure, i.e., separating the symmetries of the particle-index pseudospace and the coordinate space can be carried out for any multicluster configuration, too, including the limiting case of the $A$ clusters (where $A$ is the mass number of the nucleus). Therefore, the prescription for the construction of symmetric Hamiltonians is applicable also for multicluster configurations, up to the shell-model limit.

The dynamical symmetries of the binary configurations are obtained as projections from the dynamical symmetry of the underlying ternary configuration. The clusterization $c$ is specified by a constraint on quantum number $n_{t_{c}}$ (or in the notation of the group chain (5), $m_{p} \equiv m_{c}$ ), while that of configuration $d$ fixes $n_{t_{d}}\left(m_{p} \equiv m_{d}\right)$. (In the example, ${ }^{16} \mathrm{O}+{ }^{8} \mathrm{Be}: n_{t_{c}}=4, m_{c}=4 ;{ }^{20} \mathrm{Ne}+{ }^{4} \mathrm{He}: n_{t_{d}}=8, m_{d}=0$.) These quantities, $n_{t_{c}}$ and $n_{t_{d}}$ (or $m_{c}$ and $m_{d}$ ), are not good quantum numbers at the same time. The basis state with a definite $n_{t_{c}}$ is a finite linear combination of those of $n_{t_{d}}$, and vice versa. Therefore, the two-channel MUSY is a partial symmetry [18] in the sense that not each of the quantum numbers is valid in the two-channel multiplets. But it is remarkable that the pseudospin-scalar Hamiltonian is diagonal in both bases (independent of $m_{p}$ ); i.e., a configuration-independent interaction can be constructed.

The projection to the binary configurations takes place in the following way. (i) The ternary Hamiltonian of Eq. (9) contains a simple form of the $F(\lambda, \mu, L)$ function: $C_{\mathrm{SU}(3)}^{(2)}$. Since $F(\lambda, \mu, L)$ corresponds to a pure shell-model contribution (it contains no relative motion, i.e., $n_{R}$ dependence), other functions of this kind would behave in the same way (they are invariant with respect to the transformations from ternary to binary configurations). (ii) The relative motion quantum number $n_{s_{c}}$ or $n_{s_{d}}$ of the binary configurations is obtained from the $N_{6}=n_{s}+n_{t}$ ternary quantum numbers, by fixing $n_{t}$ for the configurations $c$ and $d$, respectively (in our example $\left.n_{t_{c}}=4, n_{t_{d}}=8\right)$. Then the ternary Hamiltonians for the Jacobi coordinates $c$ and $d$ are

$$
\begin{aligned}
& H_{c}=(\epsilon+4 \gamma)+\gamma n_{s_{c}}+\delta C_{\mathrm{SU}(3)}^{(2)}+(\beta+4 \theta) L^{2}+\theta n_{s_{c}} L^{2}, \\
& H_{d}=(\epsilon+8 \gamma)+\gamma n_{s_{d}}+\delta C_{\mathrm{SU}(3)}^{(2)}+(\beta+8 \theta) L^{2}+\theta n_{s_{d}} L^{2} .
\end{aligned}
$$


Note that the parameters $\epsilon, \gamma, \delta, \beta$, and $\theta$ do not depend on the index $c$ or $d$; they belong to the pseudospin-invariant ternary Hamiltonian. However, the parameters of Eq. (2), i.e., the energy formula of the binary configurations, are different for clusterization $c$ and $d: \epsilon_{c}, \gamma_{c}, \ldots$ and $\epsilon_{d}, \gamma_{d}, \ldots$ Calculating the eigenvalues of the projected ternary Hamiltonians of Eq. (7) according to Eq. (2), one arrives at the relations $\gamma_{c}=\gamma_{d}=$ $\gamma, \epsilon_{c}=\epsilon_{d}-4 \gamma, \theta_{c}=\theta_{d}=\theta, \beta_{c}=\beta_{d}-4 \theta, F_{c}(\lambda, \mu, L)=$ $F_{d}(\lambda, \mu, L)$; i.e., the projections from the underlying ternary dynamical symmetry result exactly in the relations of the binary dynamical symmetries, which were obtained from the phenomenological introduction of the MUSY [5].

Similarly to the Hamiltonian, some other operators, e.g., those of the electric quadrupole and magnetic dipole transitions, are also diagonal in both bases.

So far we discussed the relative motion part of the cluster configurations. When there is (at least one) non-SU(3)-scalar cluster in the ternary configuration, then $n_{\mathrm{C}}, L_{\mathrm{C}}$, and $Q_{\mathrm{C}}$ also contribute to the operators. Then the corresponding group chain is $\mathrm{U}_{\mathrm{C}}(3) \otimes\left\{\mathrm{U}_{\mathrm{R}}(7) \supset \mathrm{U}_{\mathrm{R}}(1) \otimes \mathrm{U}_{\mathrm{R}}(6)\right\} \supset$ $\left\{\mathrm{U}_{\mathrm{C}}(3) \otimes \mathrm{U}_{\mathrm{R}}(3) \supset \mathrm{U}(3) \supset \mathrm{SU}(3) \supset \mathrm{SO}(3) \supset \mathrm{SO}(2)\right\} \otimes$ $\left\{\mathrm{U}_{p}(2) \supset \mathrm{SU}_{p}(2) \supset \mathrm{SO}_{p}(2)\right\}$. Here $\mathrm{U}_{\mathrm{C}}(3)$ stands for the internal symmetry group of the nonscalar cluster, if there is only a single one, and it stands for the coupled symmetry of the internal structures if there are more nonscalar clusters.

Comparing the multichannel symmetry with the dynamical supersymmetry, the following can be said. Both the SUSY and the MUSY are composite symmetries; they describe a coupled system with two components: bosonic and fermionic in the SUSY and different cluster configurations in the MUSY. Both components have a dynamical symmetry, and some extra transformations connect them, by taking particles from one sector to the other. They are the supertransformation in the former case, and Talmi-Moshinsky transformations in the latter case. The SUSY preserves all the quantum numbers, and thus provides a supersymmetric multiplet, while the MUSY is a partial symmetry: one quantum number is not valid for both channels at the same time. Nevertheless, it is very interesting that the present mathematical foundation of the MUSY shows how the configuration-independent cluster-cluster interactions can be constructed, which are really invariant with respect to the pseudospin transformations. From this viewpoint the MUSY is more strict; in the SUSY models the interactions are usually not invariant with respect to the supertransformations [due to the fact that the first subgroup of their chain is already a (direct product) Lie group; i.e., its Casimir operator is not necessarily invariant with respect to the supertransformations]. In this respect the MUSY is more similar to the SUSY models of the particle physics [19], where this kind of invariance is usual, whereas in the nuclear SUSY models it is exceptional.

To sum up, we showed that a multichannel dynamical symmetry, which connects different cluster configurations in a nucleus, can be derived from the dynamical symmetry of an underlying multicluster configuration. It establishes a strict correlation between the observables of different clusterizations; e.g., the Hamiltonian of one cluster configuration may completely determine the energy spectrum of another cluster configuration. The first tests of the MUSY, performed after the phenomenological introduction [5,20], seem to be promising, but much work remains to be done in order to check how well this new symmetry is realized in nuclear spectra.

This work was supported by the OTKA (Grants No. K72357 and No. K106035), and by the JSPS-MTA Bilateral Project (No. 119). One of the authors (J.Cs.) acknowledges the hospitality of the Hokkaido University, where a part of this work was completed.
[1] E. P. Wigner, Phys. Rev. 51, 106 (1937).

[2] F. Iachello and P. Van Isacker, The Interacting Boson-Fermion Model (Cambridge University Press, Cambridge, UK, 1991).

[3] A. Frank, J. Jolie, and P. Van Isacker, Symmetries in Atomic Nuclei (Springer, New York, 2009).

[4] G. Lévai, J. Cseh, and P. Van Isacker, Eur. Phys. J. A 12, 305 (2001); J. Phys. G 34, 1729 (2007).

[5] J. Cseh, Phys. Rev. C 50, 2240 (1994).

[6] J. P. Elliott, Proc. R. Soc. London Ser. A 245, 128 (1958).

[7] F. Iachello and A. Arima, The Interacting Boson Model (Cambridge University Press, Cambridge, UK, 1987).

[8] D. J. Rowe, Rep. Prog. Phys. 48, 1419 (1985).

[9] J. Cseh, Phys. Lett. B 281, 173 (1992); J. Cseh and G. Lévai, Ann. Phys. (NY) 230, 165 (1994).

[10] V. Bargmann and M. Moshinsky, Nucl. Phys. 18, 697 (1960).
[11] B. Kaufman and C. Noack, J. Math. Phys. 6, 142 (1965).

[12] A. Gal, Ann. Phys. (NY) 49, 341 (1968).

[13] H. Horiuchi, Prog. Theor. Phys. 58, 204 (1977).

[14] F. Iachello, Phys. Rev. C 23, 2778 (1981); F. Iachello and R. D. Levine, J. Chem. Phys. 77, 3046 (1982).

[15] H. Horiuchi, Prog. Theor. Phys. Suppl. 62, 90 (1977).

[16] F. Iachello, J. Cseh, and G. Lévai, Acta Phys. Hung. New Ser.: Heavy Ion Phys. 1, 91 (1995).

[17] R. Bijker, F. Iachello, and A. Leviatan, Ann. Phys. (NY) 236, 69 (1994).

[18] P. Van Isacker, Phys. Rev. Lett. 83, 4269 (1999).

[19] R. V. Jolos and P. von Brentano, Phys. Rev. C 60, 064318 (1999).

[20] J. Cseh, G. Lévai, A. Ventura, and L. Zuffi, Phys. Rev. C 58, 2144 (1998). 